\title{
AMCoR
}

Asahikawa Medical University Repository http://amcor.asahikawa-med.ac.jp/

International Journal of Hematology (2016.1) 103(1):34-43.

The three isoforms of hepcidin in human serum and their processing determined by liquid chromatography-tandem mass spectrometry (LCtandem MS)

Lynda Addo, Katsuya Ikuta, Hiroki Tanaka, Yasumichi Toki, Mayumi Hatayama, Masayo Yamamoto, Satoshi Ito, Motohiro Shindo, Yusuke Sasaki, Yasushi Shimonaka, Mikihiro Fujiya, Yutaka Kohgo 


\section{The Three Isoforms of Hepcidin in Human Serum and their Processing Determined by Liquid Chromatography-tandem Mass Spectrometry (LC-tandem MS)}

Lynda Addo $^{1}$, Katsuya Ikuta ${ }^{1}$, Hiroki Tanaka ${ }^{2}$, Yasumichi Toki ${ }^{1}$, Mayumi Hatayama ${ }^{1}$, Masayo Yamamoto ${ }^{1}$, Satoshi Ito ${ }^{1}$, Motohiro Shindo ${ }^{1}$, Yusuke Sasaki ${ }^{3}$, Yasushi Shimonaka ${ }^{3}$, Mikihiro Fujiya ${ }^{1}$, Yutaka Kohgo ${ }^{1,2}$

Running head: Three isoforms of hepcidin in human serum

Type of article: Original Article

${ }^{1}$ Division of Gastroenterology and Hematology/Oncology, Department of Medicine, Asahikawa Medical University, 2-1-1-1, Midorigaoka-Higashi, Asahikawa, Hokkaido 078-8510, Japan.

${ }^{2}$ Department of Gastrointestinal Immunology and Regenerative Medicine, Asahikawa Medical University, 2-1-1-1, Midorigaoka-Higashi, Asahikawa, Hokkaido 078-8510, Japan.

${ }^{3}$ Product Research Department, Kamakura Research Labs, Chugai Pharmaceutical Co., Ltd., 200 Kajiwara, Kamakura, Kanagawa, 247-8530, Japan.

*Corresponding Author: Katsuya Ikuta, M.D, Ph.D

Division of Gastroenterology and Hematology/Oncology, Department of Medicine, Asahikawa Medical University. 2-1-1-1 Midorigaoka-Higashi, Asahikawa 078-8510. Hokkaido, Japan.

Telephone: +81-166-68-2462

Fax:+ 81-166-68-2469

E-mail: ikuta@asahikawa-med.ac.jp 


\section{ABSTRACT}

Hepcidin, the iron regulatory hormone, has three isoforms; -20, -22 and -25. While hepcidin-25 has been studied extensively, the physiological significance of other isoforms remains poorly understood. Using a quantitative method based on liquid-chromatography-tandem mass spectrometry (LC-tandem MS) developed by our group, we quantified hepcidin isoforms in human serum to elucidate their characteristics, and investigated the role of hepatocytes in isoform processing. Hepcidin isoforms in serum obtained from 40 healthy volunteers were quantified. Synthetic hepcidin peptides were added to healthy serum, and to HepG2 culture media, and hepcidin isoform concentrations determined. All three hepcidin isoforms were detected in human serum; however, hepcidin-25 concentrations were highest. The three hepcidin isoforms showed a strong positive correlation with each other and with serum ferritin. Additionally, while hepcidin-20 was strongly correlated with serum creatinine, the other isoforms were not. Hepcidin-20 and -25 levels were also increased in chronic kidney disease (CKD) serum. Hepcidin-22 rapidly degraded into hepcidin-20, whereas hepcidin-25 remained relatively stable. Finally, hepcidin-22 degradation into hepcidin-20 was accelerated in the presence of HepG2. This method has enabled us to reveal fundamental characteristics of the three hepcidin isoforms in serum and may be a powerful tool for quantifying hepcidin isoform expression and processing.

Keywords: Hepcidin, hepcidin isoforms, LC-tandem MS 


\section{INTRODUCTION}

Balancing systemic iron levels within narrow limits is critical for human health, as diseases of iron deficiency and iron overload belong to the most frequent disorders worldwide [1, 2]. Previous studies have identified the small peptide hormone hepcidin as the key regulator of systemic iron metabolism [3-7]; it inhibits iron export from the cell by internalizing and degrading ferroportin [8, 9]. In accordance with this mechanism, chronically elevated hepcidin levels result in decreased iron, such as occurs in anaemia of chronic disease (ACD), whereas inappropriately low levels of hepcidin result in iron overload, such as in hereditary hemochromatosis (HH) [10].

In humans, hepcidin is synthesized in the liver as an 84 amino acid pre-propeptide; it undergoes sequential proteolytic cleavage to form the 25-amino acid bioactive hormone, hepcidin-25, which is secreted in plasma. Subsequent amino-terminal processing of the 25 amino acid isoform results in the production of two smaller isoforms, hepcidin -20 and -22, whose physiological roles remain unclear. Both smaller isoforms have been reported to be present in urine, but not, or at very low concentrations, in serum [7, 11]. Moreover, while hepcidin-25 has been extensively studied, systematic investigations into the smaller isoforms in human disease are still lacking. Available reports however suggest that hepcidin-20, at acidic $\mathrm{pH}$ ( $\mathrm{pH}$ 5.0), may possess greater antimicrobial and fungicidal activity than hepcidin-25, but no capability to bind ferroportin [12, 13].

Quantifying hepcidin in biologic fluids has therefore stimulated interest, regarding the potential applicability of this measurement as a more informative marker of iron status, compared to the traditionally known indices of iron measurement, such as serum ferritin and transferrin saturation. Until recently, assays for measuring hepcidin have lacked precision, accuracy, and internal validation; however, over the last few years, several reliable hepcidin assays that address these limitations have become available, some of which include radioimmunoassay, Enzyme Linked Immunosorbent Assay (ELISA), ProteinChip Array and the even more popular Surface Enhanced Laser Desorption/Ionization Mass Spectrometry (SELDI-TOF MS) [14-21]. These proteomic approaches are well-known to have the potential of evaluating not only the predominant 25-amino acid isoform, but also the shorter ones. To date however, considerable differences continue to exist among the different hepcidin measurement methods, some of which may be attributed to the different values various laboratories assign to the internal and external standards used. 
In this study, we used an LC-tandem MS method we had previously established to measure the three hepcidin isoforms in serum obtained from healthy volunteers at the Asahikawa Medical University. This LC-tandem MS method was useful because it is able to measure all three hepcidin isoforms in serum simultaneously [22]. We also investigated how the three hepcidin isoforms are processed, as well as the role hepatocytes may play in hepcidin processing. 


\section{MATERIALS AND METHODS}

\section{Ethics}

Approval for this study was obtained from the Ethics Review Committee of the Asahikawa Medical University. Informed consent was also obtained from all the study participants.

\section{Subjects}

Forty healthy volunteers from the Asahikawa Medical University were randomly recruited to participate in this study. Commercially obtained healthy (Uniglobe Research Corporation, Reseda, CA 91335, U.S.A.) and chronic kidney disease (CKD) serum samples (ProteoGenex, Inc., Culver City, CA 90230) were also included in this study. All donors of CKD serum were receiving dialysis without iron administration.

\section{Hepcidin quantification by LC-tandem MS method}

Standards for human hepcidin-20, -22, and -25 were synthesized; trichloracetic acid (TCA) precipitation was then performed to rid the serum samples of large molecules, after which hepcidin isoforms in the supernatant were quantified. The pre-treated samples were applied to the high-performance liquid chromatograph to separate hepcidin from other proteins, and then sent to a mass spectrometer to quantify each hepcidin isoform separately and simultaneously by settling selected reaction monitoring (SRM) transitions and collision energies for each isoform respectively [22, 23]. In the experiments to investigate hepcidin processing, synthetic hepcidin peptides were added to healthy serum obtained from Uniglobe Research Corporation (Reseda, CA, U.S.A.); $1 \mu \mathrm{L}$ of synthetic hepcidin-22 or -25 peptides $(10 \mu \mathrm{g} / \mu \mathrm{L})$ were added to $99 \mu \mathrm{L}$ of each serum sample in a 96 -well plate to make a final concentration of $100 \mathrm{ng} / \mathrm{mL}$, and using our LC-tandem MS method, the hepcidin isoform concentration in each of the samples were determined after $0,1,3,6,12,24,30,48$ and 72 hours incubation at $37^{\circ} \mathrm{C}$. To investigate the stabilities of the standard hepcidin peptides in the absence of serum, $100 \mathrm{ng} / \mathrm{mL}$ of each standard peptide (hepcidin-20, -22, or -25) was incubated in $100 \mu \mathrm{L}$ of phosphate buffered saline containing $0.02 \%$ Tween 20 without serum at $37^{\circ} \mathrm{C}$ for 72 hours. After incubation, the concentrations of the three hepcidin isoforms were measured using the LC-tandem MS method. 


\section{Biochemical analysis for serum samples}

Blood samples obtained from all the study subjects were analyzed for complete blood count, serum iron, serum ferritin, total iron binding capacity (TIBC), blood urea nitrogen (BUN), creatinine, aspartate aminotransferase (AST) and alanine aminotransferase (ALT).

\section{Cell line}

The human hepatocellular carcinoma cell line, HepG2, was obtained from ATCC (Rockville, MD, USA). These cells were cultured in DMEM (Wako, Tokyo, Japan) and supplemented with 10\% FCS and penicillin-streptomycin (Wako). To investigate the role of hepatocytes in hepcidin processing, synthetic hepcidin-20, -22 and -25 peptides were added to culture media with and without HepG2 to a final concentration of $100 \mathrm{ng} / \mathrm{mL}$, and incubated for 48 hours at $37^{\circ} \mathrm{C}$; the supernatant medium was then collected and the hepcidin isoform concentrations analyzed.

\section{Statistical analysis}

Statistical analysis of the three hepcidin isoforms were performed using Bonferroni's multiple comparison test, and $\mathrm{p}<0.05$ was considered to be statistically significant. Also, Pearson's R Correlation test was used to determine correlation between the hepcidin isoforms, as well as correlation of biochemical markers with the hepcidin isoforms. $|\mathrm{R}|>0.7$ was considered to be strongly correlated while a $P$ value $<0.05$ (two tailed) was considered statistically significant. 


\section{RESULTS}

\section{Determination of the three isoforms of hepcidin in human serum}

Figure 1A shows the concentrations of the three hepcidin isoforms in all 40 serum samples analyzed; hepcidin-20 $($ mean $=2.636 \mathrm{ng} / \mathrm{mL}),-22($ mean $=0.973 \mathrm{ng} / \mathrm{mL})$ and $-25($ mean $=12.401 \mathrm{ng} / \mathrm{mL})$. Compared to the two other isoforms, hepcidin-25 levels were present in the highest concentrations, and in statistically significant amounts (Figure 1A). It is also important to note that the concentrations of the three isoforms varied considerably among the individual samples (Figure 1B, 1C).

\section{Correlation among hepcidin isoforms:}

A highly positive correlation was observed between the three hepcidin isoforms [hepcidin-20 and hepcidin-22 ( $\mathrm{R}=0.8686, P<0.0001)$, hepcidin-22 and hepcidin-25 $(\mathrm{R}=0.8218, P<0.0001)$, and hepcidin-20 and hepcidin-25 ( $\mathrm{R}=0.7426, P<0.0001)]$ (Figure 2A, 2B, 2C).

\section{Correlation between serum hepcidin and serum ferritin:}

We focused on the correlation between all three hepcidin isoforms and biomarkers (red blood cell, hemoglobin, hematocrit, serum iron, TIBC and serum ferritin) which are associated with body iron metabolism. All three isoforms correlated positively with serum ferritin [hepcidin-20 and ferritin $(\mathrm{R}=$ 0.8394), hepcidin-22 and ferritin $(\mathrm{R}=0.8127)$, hepcidin-25 and ferritin $(\mathrm{R}=0.7680)]$ (Figure 3$)$. However, the isoforms did not show strong correlation with the other iron biomarkers (Table 1).

\section{Correlation between hepcidin- 20 and serum creatinine (Cre)}

We then evaluated the correlation between the hepcidin isoforms and other routinely analyzed serum biomarkers in healthy volunteers, such as BUN, creatinine, ALT and AST. Hepcidin-20 showed a positive correlation with serum creatinine $(\mathrm{R}=0.7345)$ (Figure $4 \mathrm{~A})$, but no other strong correlations were observed (Table 2). Because there was a positive correlation between hepcidin-20 and serum creatinine in healthy volunteers, we then evaluated the correlation between hepcidin and creatinine in CKD serum and found that higher levels of hepcidin-20 (mean $=28.454 \mathrm{ng} / \mathrm{mL})$ and $-25(\mathrm{mean}=$ $46.752 \mathrm{ng} / \mathrm{mL}$ ) were present in CKD serum (Figure 4B) than in healthy serum. 


\section{Hepcidin-22 is degraded into hepcidin-20 in serum}

We then evaluated the stability of the three hepcidin isoforms in serum. Synthetic hepcidin-22 and -25 peptides were added to commercially obtained healthy human serum as indicated in the Materials and methods section, and the concentrations of the three hepcidin isoforms measured at each time point. When synthetic hepcidin-22 was added to serum, its levels decreased to almost $6 \%$ in 72 hrs, whereas hepcidin-20 levels increased to $83.216 \pm 4.789 \mathrm{ng} / \mathrm{mL}$; this suggests that the high dose $(100 \mathrm{ng} / \mathrm{mL})$ hepcidin-22 peptide was simultaneously degraded into hepcidin-20 in serum (Figure 5A). On the other hand, when synthetic hepcidin-25 was added to serum, its levels only decreased by about $70 \%$ in 72 hrs (Figure 5B), indicating that hepcidin-25 was more stable than hepcidin-22 in serum. Synthetic hepcidin isoforms in serum-free buffer however remained substantially stable during the 72 hour incubation period; $93.6 \pm 1.3 \%$ of hepcidin-20, $86.6 \pm 3.3 \%$ of hepcidin-22, and $89.1 \pm 4.4 \%$ of hepcidin-25 were measurable after the incubation period, with no detection of the smaller isoforms from hepcidin-22 or hepcidin-25 (Table 3). Additionally, no substantial difference in hepcidin-22 and hepcidin-25 processing was observed when the experiment was performed using CKD serum (data not shown).

\section{Hepcidin-22 is degraded to hepcidin-20 in HepG2 culture}

Because hepatocytes are the main source of hepcidin, we hypothesized that hepcidin processing may be accelerated by hepatocytes, and thus assessed the stability of each hepcidin isoform using the human hepatocellular carcinoma cell line, HepG2. Only slight changes in the hepcidin isoforms were observed when each hepcidin peptide was cultured in culture medium alone (Figure 6A), however, when hepcidin-22 peptides were incubated in HepG2 cell culture medium, hepcidin-22 levels were found to be reduced to hepcidin-20 after 48 hours incubation (Figure 6B). 


\section{DISCUSSION}

Ever since its discovery, many research laboratories have continued to devise techniques to identify hepcidin and its isoforms in biologic fluids, such as in urine and serum. In this study, our previously reported LC-tandem MS method $[22,23]$ was confirmed as valuable for the quantification of hepcidin in serum, not only with respect to the main isoform, hepcidin-25, but also to hepcidin-20 and -22. With this method, all three isoforms of hepcidin were detected in human serum, although notable variations in the isoforms were also observed (Fig. 1). Hepcidin-25 was found to be present in the highest concentrations; thus from the view of quantity, hepcidin-25 can be described as the main hepcidin isoform in healthy serum, consistent with the widely accepted view that hepcidin-25 is the major isoform of hepcidin [6]. Factors such as serum ferritin, which affect hepcidin expression, and whose levels are varied even in health, may account for the considerable variation in hepcidin-25 observed in healthy serum. In the future, it would be important to establish a strict and clear criteria for defining the healthy subject group, as this would be helpful in establishing a reliable reference range for hepcidin-25 in health.

In addition to factors that may affect hepcidin expression in healthy persons, an acute and chronic pro-inflammatory state exists in patients with CKD, which contributes to iron retention and inhibition of iron utilization, as well as difficulties in hepcidin peptide filtration in the kidney and the presence of uremic substances in serum. It may therefore be useful to evaluate hepcidin-20 and 25 levels in the urine of patients with CKD in future studies, as this may provide valuable information regarding the significance of increased hepcidin-20 levels in CKD. Also, since the CKD serum evaluated in this study were only obtained from patients undergoing dialysis, it would be useful to investigate CKD patients not undergoing dialysis in the future, to elucidate whether renal dysfunction itself affects hepcidin expression. The strong positive correlation observed among the hepcidin isoforms implies that hepcidin-25 may be degraded to produce the smaller isoforms (hepcidin-20 and -22). These correlations however do not exclude the possibility that hepcidin-20 and hepcidin-22 may be directly generated from pro-hepcidin by convertases [11, 24]. A stable and reliable method for simultaneously quantifying the 3 hepcidin isoforms, and pro-hepcidin in serum would be useful and ideal to help elucidate the dynamics of hepcidin metabolism in the human body. It would also be important to further improve our LC-tandem MS-based hepcidin quantification system to be applicable for pro-hepcidin 
quantification, although establishing pro-hepcidin standard peptides will not be easy. In normal iron homeostasis, increased plasma transferrin saturation raises hepcidin levels [25-29], which causes the iron exporter ferroportin to be internalized and degraded, limiting further intestinal iron absorption and release from macrophages [6, 30]. However, under pathological conditions, where hepcidin synthesis is inadequate, ferroportin expression is excessive, and iron entry into the circulation from the gastrointestinal tract and macrophages is increased. In this study, serum hepcidin was found to correlate positively with serum ferritin, a finding that is compatible with other reports [31, 32]. Also, while hepcidin-20 was quantifiable in almost all the samples we analyzed (39 out of 40 samples), Campostrini and her group have in 2 separate studies, using SELDI-TOF-MS, reported detecting hepcidin-20 in 62\% (35 out of 57) [18] and 54.2\% (854 out of 1577 subjects) [33] of the general population. These findings, together with the results of our study, strongly suggest that the relative contribution of hepcidin-20 to total serum hepcidin is not negligible at the population level, and clearly contradicts the simplistic view that hepcidin-20 is merely a catabolic by-product of hepcidin-25. Additionally, we found extremely high levels of hepcidin-20 in kidney disease. Using the WCX-TOF-MS assay, Kroot and colleagues compared data from 23 healthy controls with several groups of patients with heterogeneous disorders of iron metabolism and found hepcidin-20 to be frequently increased in several diseases, particularly in CKD [34]. In addition, Suzuki et al also reported elevated levels of hepcidin-20 during the acute phase of myocardial infarction [35]. These findings demonstrate the possible usefulness of hepcidin-20 as a biomarker of disease in the future. A recent study also reports identifying a novel isoform, hepcidin-24, in kidney disease. However, they report that this isoform, like the -22 and -20 isoforms, is a significantly less potent inducer of ferroportin degradation compared to hepcidin-25 [36].

To date, very little is known about the biological roles or the mechanism by which the smaller isoforms of hepcidin (the 22 and 20 polymer peptides) are processed. In this study, hepcidin-22 was found to be degraded to hepcidin-20 in both healthy and CKD serum. However, synthetic hepcidin isoforms exhibited relative stability in the absence of serum, despite slight decreases in peptide concentrations (Table 3). Further, we did not detect the smaller hepcidin isoforms from hepcidin-22 or hepcidn-25 in this experiment, indicating that the degradation of hepcidin-22 or hepcidin-25 into the smaller isoforms occurs in serum.

We then hypothesized that hepcidin-25 breakdown will likely be increased in CKD; however, 
the stability of hepcidin-25 was unchanged in both healthy and CKD serum, implying that the increase in hepcidin-20 in CKD was unlikely to be as a result of hepcidin-25 processing, but possibly by an unknown mechanism.

Furthermore, while hepcidin-20 concentration increased, hepcidin-22 levels were observed to decrease when synthetic hepcidin-22 was added to HepG2 cells; this indicates that the processing of hepcidin-22 to hepcidin-20 may have been accelerated in the presence hepatocytes. This also suggests that the influence of endogeneously expressed hepcidin from HepG2 may be significant, as the cell culture medium only contained hepcidin-22 and -25 in our previous study [22]. It is entirely possible though that hepcidin-20 was present in that experiment, but beyond the detection limit of our procedure, hence our inability to measure it. In the present study, synthetic hepcidin peptides were added in amounts much higher than occurred endogeneously. For example, mean serum hepcidin concentration in healthy persons, which implies endogenous secretion of hepcidin-22 and -25, were found to be only $0.973 \mathrm{ng} / \mathrm{mL}$ and $12.401 \mathrm{ng} / \mathrm{mL}$ respectively, but in the present study, hepcidin-22 and -25 were added at $100 \mathrm{ng} / \mathrm{mL}$ concentrations. Thus the observations made in this experiment mainly showed the degradation of spiked hepcidin-22 or -25, which implies that the contribution of secreted hepcidin to the total hepcidin concentration was small. Another explanation for this is that, if the contribution of the individal endogeneous hepcidin isoforms to the total hepcidin concentration were large, the concentration of each of the hepcidin isoforms should have increased with time, which did not occur.

Considering that hepcidin is mainly produced by the liver, it is not surprising that hepatocytes may likely play an important role in the breakdown of hepcidin into its various isoforms. Moreover, hepatocytes are the target cells of hepcidin, as hepatocytes express ferroportin and play a role in physiologic iron supply in response to body iron demands. Thus the liver may function not only in iron sequestration, but also in iron regulation by fine tuning hepcidin expression during both hepcidin production and processing. Finally, while the mechanism involved in the processing of hepcidin and its isoforms is quite evidently a complex one, we were able to elucidate a part of this process in this study.

\section{CONCLUSION}

Although hepcidin is now acknowledged to be the main iron regulatory hormone, several aspects of this hormone, such as the mechanism by which it is produced, degraded and filtered from the 
kidney, remains to be elucidated. The results of the present study have provided some fundamental information about the hepcidin isoforms. We have also showed that one of the hepcidin isoforms may be a valuable biomarker for CKD, indicating the possible usefulness of the hepcidin isoforms in other haematological diseases. Additionally, our quantification method may be a powerful tool for research regarding hepcidin and its isoforms. Further research on the hepcidin isoforms in other diseases, with a larger sample size may also reveal other possible usefulness of hepcidin in the future.

\section{Conflict of Interest:}

Lynda Addo, Katsuya Ikuta, Yasumichi Toki, Mayumi Hatayama, Masayo Yamamoto, Satoshi Ito, Motohiro Shindo, Mikihiro Fujiya, Yutaka Kohgo (Department of Gastroenterology and Hematology/Oncology, Division of Medicine, Asahikawa Medical University) and Hiroki Tanaka (Department of Gastrointestinal Immunology and Regenerative Medicine, Asahikawa Medical University) received research funding from Chugai Pharmaceutical Co. Ltd. This study was also performed in collaboration with Chugai Pharmaceutical Co. Ltd. These departments also received collaborative research funding for research work concerning iron metabolism from Novartis Pharma K.K. and Asahi Kasei Medical Co. Ltd. 


\section{REFERENCES}

1. Aisen P, Enns C, Wessling-Resnick M. Chemistry and biology of eukaryotic iron metabolism. Int J Biochem Cell Biol. 2001; 33:940-59.

2. Andrews NC. Disorders of iron metabolism. N Engl J Med. 1999; 341:1986-95.

3. Pigeon C, Ilyin G, Courselaud B, Leroyer P, Turlin B, Brissot P et al. A new mouse liver-specific gene, encoding a protein homologous to human antimicrobial peptide hepcidin, is overexpressed during iron overload. J Biol Chem 2001; 276:7811-19.

4. Nicolas G, Viatte L, Bennoun M, Beaumont C, Kahn A, Vaulont S. Hepcidin, a new iron regulatory peptide. Blood Cells Mol Dis. 2002; 29:327-35.

5. Ganz T. Hepcidin, a key regulator of iron metabolism and mediator of anemia of inflammation. Blood. 2003; 102:783-88.

6. Krause A, Neitz S, Magert HJ, Schultz A, Forssmann WG, Schultz-Knappe P, et al. LEAP-1, a novel highly disulfide-bonded human peptide, exhibits antimicrobial activity. FEBS Lett. 2000; 480:147-50.

7. Park CH, Valore EV, Waring AJ, Ganz T. Hepcidin, a urinary antimicrobial peptide synthesized in the liver. J Biol Chem. 2001; 276: 7806-10.

8. Nemeth E, Tuttle MS, Powelson J, Vaughn MB, Donovan A, Ward DM et al. Hepcidin regulates cellular iron efflux by binding to ferroportin and inducing its internalization. Science. 2004; 306: 2090-93.

9. De Domenico I, Nemeth E, Nelson JM, Phillips JD, Ajioka RS, Kay MS et al. The hepcidin-binding site on ferroportin is evolutionarily conserved. Cell Metab. 2008; 8:146-56.

10. Mei S, Wang H, Fu R, Qu W, Xing L, Wang G, et al. Hepcidin and GDF15 in anemia of multiple myeloma.Int J Hematol. 2014; 100: 266-73.

11. Kroot JJC, Tjalsma H, Fleming RE, Swinkels DW. Hepcidin in Human Iron Disorders: Diagnostic Implications. Clinical Chemistry. 2011; 57:1650-69.

12. Maisetta G, Petruzzelli R, Brancatisano FL, Essin S, Vitali A, Campa M et al. Antimicrobial activity of human hepcidin-20 and 25 against clinically relevant bacterial strains: effect of copper and acidic pH. Peptides 2010; 31:1995-2002. 
13. Tavanti A, Maisetta G, Del Gaudio G, Petruzelli R, Sanguinetti M, Batoni G et al. Fungicidal activity of the human peptide hepcidin 20 alone or in combination with other antifungals against Candida glabrata isolates. Peptides. 2011; 32:2484-7.

14. Piperno A, Mariani R, Trombini and Girelli D. Hepcidin modulation in human diseases: From research to clinic. World J Gastroenterol. 2009; 15:538-51.

15. Kemna E, Tjalsma H, Podust VN and Swinkels DW. Mass Spectrometry-Based Hepcidin Measurements in Serum and Urine: Analytical Aspects and Clinical Implications. Clin Chem 2007; 53:620-8.

16. Ganz T, Olbina G, Girelli D, Nemeth E, Westerman M. Immunoassay for huma serum hepcidin. Blood. 2008; 112:4292-7.

17. Tomosugi N, Kawabata H, Wakatabe R, Higuchi M, Yamaya H, Umehara H et. al. Detection of serum hepcidin in renal failure and inflammation by using ProteinChip system. Blood. 2006; 108:1381-7.

18. Campostrini N, Castagna A, Zaninotto F, Bedogna V, Tessitore N, Poli A et al. Evaluation of Hepcidin Isoforms in Hemodialysis Patients by a Proteomic Approach Based on SELDI-TOF MS. J Biomed Biotechnol. 2010; 2010:329646.

19. Girelli D, De Domenico I, Bozzini C, Campostrini N, Busti F, Castagna A et al. Clinical, pathological, and molecular correlates in ferroportin disease: a study of two novel mutations. J Hepatol. 2008; 664-71.

20. Kemna E, Tjalsma H, Laarakkers C, Nemeth E, Willems H, Swinkels D. Novel urine hepcidin assay by mass spectrometry. Blood. 2005; 106:3268-70.

21. Swinkels DW, Girelli D, Laarakkers C, Kroot J, Campostrini N, Kemna EH et al. Advances in quantitative hepcidin measurements by time-of-flight mass spectrometry. PLOS One. 2008; 3:e2706.

22. Hosoki T, Ikuta K, Shimonaka Y, Sasaki Y, Yasuno H, Sato K et al. Heterogeneous expressions of hepcidin isoforms in hepatoma-derived cells detected using simultaneous LC-MS/MS. Proteomics Clin. Appl. 2009; 3:1256-64.

23. Sasaki Y, Shimonaka Y, Ikuta K, Hosoki T, Sasaki K, Torimoto Y, et al. Hepcidin production in 
response to iron is controlled by monocyte-derived humoral factors. Int J Hematol. 2014; 99: $12-20$.

24. Malyszko J. Hepcidin Assays: Ironing Out Some Details. Clin J Am Soc Nephrol. 2009; 4:1015-6.

25. Rossi E. Hepcidin-the iron regulatory hormone. Clin Biochem Rev. 2005; 26:47-9.

26. Ganz T, Nemeth E. Iron imports. IV. Hepcidin and regulation of body iron metabolism. Am J Physiol. Gastrointest Liver Physiol. 2006; 290:G199-203.

27. Leong WI, Lönnerdal B. Hepcidin, the recently identified peptide that appears to regulate iron absorption. J Nutr. 2004; 134:1-4.

28. Kemna EH, Tjalsma H, Willems HL, Swinkels DW. Hepcidin: from discovery to differential d iagnosis. Haematologica. 2008; 93:90-7.

29. Lin L, Valore EV, Nemeth E, Goodnough JB, Gabayan V, Ganz T. Iron-transferrin regulates hepcidin synthesis in primary hepatocyte culture through haemojuvelin and BMP2/4. Blood. 2007; 110:2182-9.

30. Ganz T. Hepcidin-- a regulator of intestinal iron absorption and iron recycling by macrophages. Best Pract Res Clin Haematol. 2005; 18:171-82.

31. Roe MA, Collings R, Dainty JR, Swinkels DW, Fairweather-Tait SJ. Plasma hepcidin concentrations significantly predict interindividual variation in iron absorption in healthy men. Am J Clin Nutr. 2009; 89: 1088-91.

32. Guo X, Zhou D, An P, Wu Q, Wang H, Wu A et al. Associations between serum hepcidin, ferritin and $\mathrm{Hb}$ concentrations and type 2 diabetes risks in a Han Chinese population. Br J Nutr. 2013; 110:2180-5.

33. Campostrini N, Traglia M, Martinelli N, et al. Serum levels of the hepcidin-20 isoform in a large general population: The Val Borbera study. J Proteomics. 2012; 76: 28-35.

34. Kroot JJ, Laarakkers GM, Geurts-Moespot AJ, Grebechtchikov N, Pickkers P, van Ede AE et al. Immunochemical and mass-spectrometry-based serum hepcidin assays for iron metabolism disorders. Clin Chem 2010; 56:1570-9.

35. Suzuki H, Toba K, Kato K, Ozawa T, Tomosugi N, Higuchi M et al. Serum hepcidin-20 is 
elevated during the acute phase of myocardial infarction. Tohoku J Exp Med. 2009; 218:93-8.

36. Laarakkers CMM, Wiegerinck ET, Klaver S, Kolodziejczyk M, Gille H, Hohlbaum AM et al. Improved Mass Spectrometry Assay for Plasma Hepcidin: Detection and Characterization of a Novel Hepcidin Isoform. PLOS ONE. 2013; 8:e75518. 


\section{FIGURE LEGENDS}

\section{Figure 1}

(A) Scatter plot of the three hepcidin isoforms in human serum (hepcidin-25, hepcidin-22 and hepcidin-20). Bonferroni’s multiple comparison test: ${ }^{*} \mathrm{p}<0.05$. (B) Concentration of the individual hepcidin isoforms in 40 serum samples from healthy volunteers. (C) Percentages of the various hepcidin isoforms in healthy serum. No hepcidin isoform was detected in one serum sample No. 35.

\section{Figure 2}

Graphs showing correlation among the three hepcidin isoforms in human serum: (A) Hepcidin-20 and hepcidin-22 (B) Hepcidin-25 and hepcidin-22 (C) Hepcidin-25 and hepcidin-20.

\section{Figure 3}

Graphs showing correlation between the three hepcidin isoforms and serum ferritin: (A) Serum ferritin and hepcidin-20 (B) Serum ferritin and hepcidin-22 (C) Serum ferritin and hepcidin-25

\section{Figure 4}

Graphs showing: (A) Correlation between hepcidin-20 and serum creatinine (Cre). (B) Hepcidin isoforms in chronic kidney dysfunction (CKD) serum, compared to those obtained from healthy volunteers (HV). Bonferroni's multiple comparison test: ${ }^{*} \mathrm{p}<0.05$.

\section{Figure 5}

Spike and recovery test of the three hepcidin isoforms in commercially obtained healthy human serum. Spiked synthetic peptides of all three hepcidin isoforms added to healthy serum. (A) Time course graph of spiked synthetic hepcidin-22 peptides in healthy serum. (B) Time course graph of synthetic hepcidin-25 peptides in healthy serum. 


\section{Figure 6}

Spike and recovery test of the three hepcidin isoforms in HepG2 culture. (A) Synthetic peptides of the three hepcidin isoforms added to culture medium containing 10\% FBS (without HepG2 cells) (B) Synthetic peptides of the three hepcidin isoforms added to HepG2 cells. 
Table 1. Correlation between the hepcidin isoforms and hematologic parameters and biomarkers of iron

\begin{tabular}{|c|c|c|c|c|c|c|c|}
\hline & & RBC & $\mathrm{Hb}$ & $\mathrm{Ht}$ & $\mathrm{Fe}$ & TIBC & Ferritin \\
\hline \multirow{2}{*}{ Hepcidin-20 } & $\mathrm{R}$ & 0.2377 & 0.382 & 0.3284 & 0.2303 & -0.5176 & 0.8394 \\
\hline & $P$ value & 0.1397 & 0.015 & 0.0385 & 0.1528 & 0.0006 & $<0.0001$ \\
\hline \multirow{2}{*}{ Hepcidin-22 } & $\mathrm{R}$ & 0.3642 & 0.4894 & 0.4625 & 0.295 & -0.5114 & 0.8127 \\
\hline & $P$ value & 0.0209 & 0.0014 & 0.0027 & 0.0646 & 0.0007 & $<0.0001$ \\
\hline \multirow{2}{*}{ Hepcidin-25 } & $R$ & 0.2252 & 0.3401 & 0.2981 & 0.3159 & -0.3013 & 0.7680 \\
\hline & $P$ value & 0.1623 & 0.0318 & 0.0617 & 0.0471 & 0.0588 & $<0.0001$ \\
\hline
\end{tabular}


Table 2. Correlation between hepcidin-20 and serum biomarkers

\begin{tabular}{|c|c|c|c|c|c|}
\cline { 3 - 5 } \multicolumn{2}{c|}{} & BUN & Cre & AST & ALT \\
\hline \multirow{2}{*}{ Hepcidin-20 } & $\mathrm{R}$ & 0.4838 & 0.7345 & -0.0053 & 0.1302 \\
\cline { 2 - 5 } & $P$ value & 0.0016 & $<0.0001$ & 0.9740 & 0.4233 \\
\hline \multirow{2}{*}{ Hepcidin-22 } & $\mathrm{R}$ & 0.4268 & 0.5096 & -0.0164 & 0.1168 \\
\cline { 2 - 6 } & $P$ value & 0.006 & 0.0008 & 0.9201 & 0.4730 \\
\hline \multirow{2}{*}{ Hepcidin-25 } & $\mathrm{R}$ & 0.3501 & 0.4383 & 0.1800 & 0.2172 \\
\cline { 2 - 6 } & $P$ value & 0.0268 & 0.0047 & 0.2664 & 0.1783 \\
\hline
\end{tabular}


Table 3. Relative stability of the synthetic hepcidin isoforms in the absence of serum

\begin{tabular}{|c|c|c|c|}
\hline \multirow{2}{*}{$\begin{array}{c}\text { Incubated synthetic } \\
\text { peptide }(\text { each } 100 \\
\text { ng/mL) }\end{array}$} & \multicolumn{3}{|c|}{$\begin{array}{r}\text { Recovery of hepcidin isoforms after incubation at } 37^{\circ} \mathrm{C} \text { for } 72 \text { hours without } \\
\text { serum (\% of initially added peptide) }\end{array}$} \\
\cline { 2 - 4 } & Hepcidin-20 & Hepcidin-22 & Hepcidin-25 \\
\hline Hepcidin-20 & $93.6 \pm 1.3$ & 0 & 0 \\
\hline Hepcidin-22 & 0 & $86.6 \pm 3.3$ & 0 \\
\hline Hepcidin-25 & 0 & 0 & $89.1 \pm 4.4$ \\
\hline
\end{tabular}




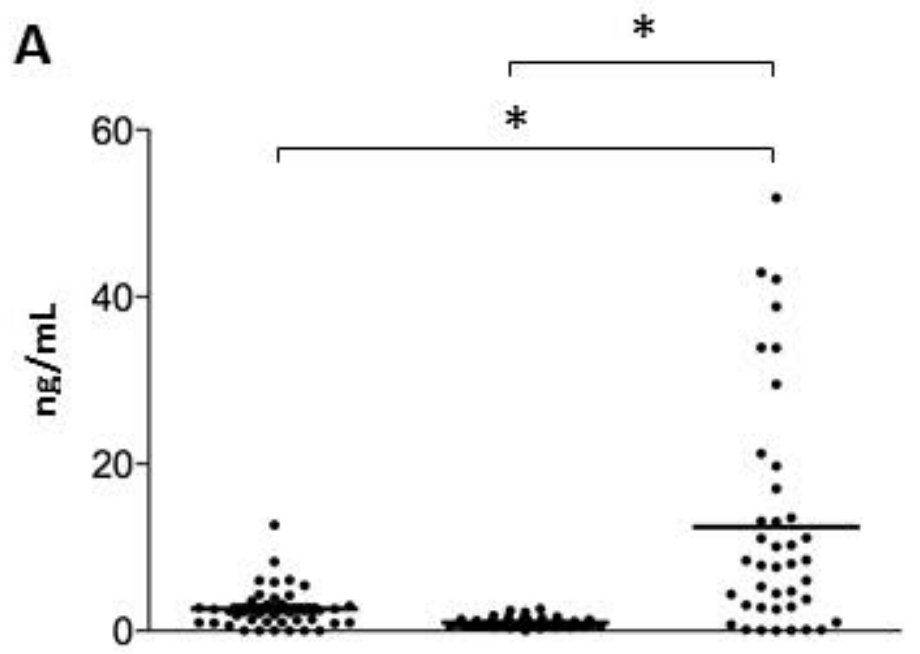

hepcidin-20 hepcidin-22 hepcidin-25

B

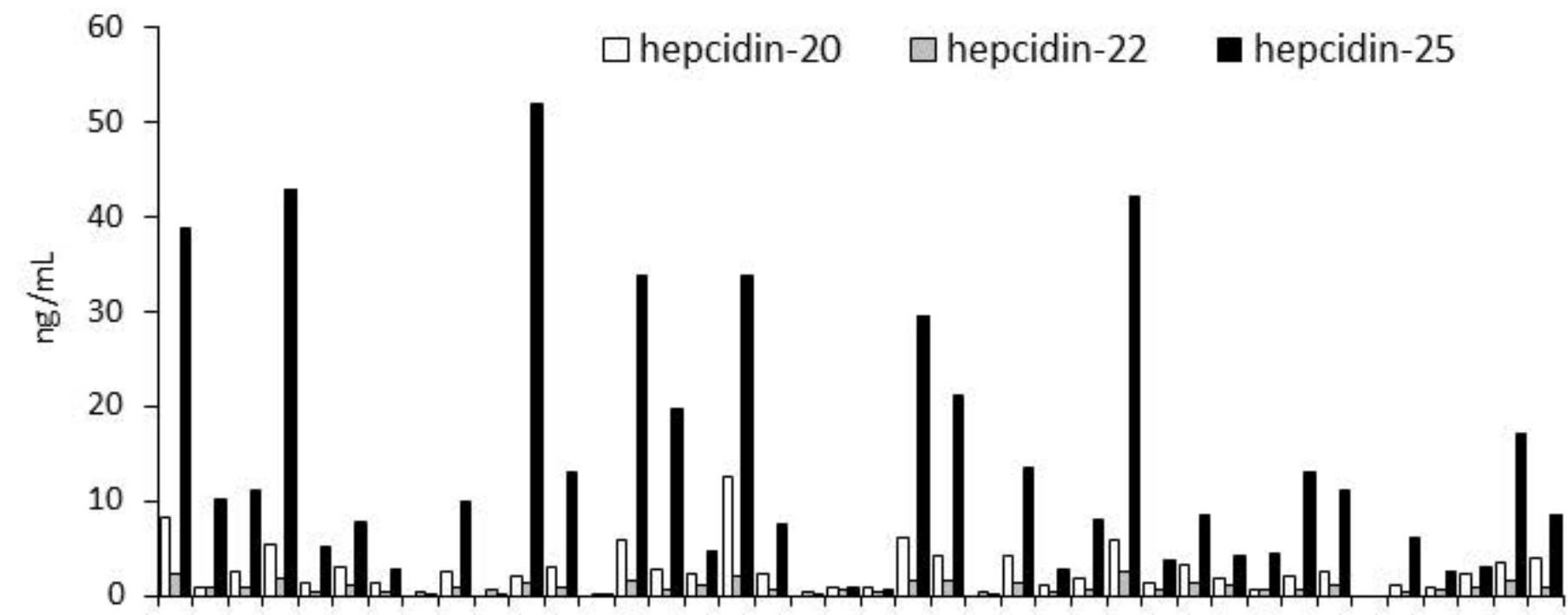

123445678910111213141516171819202122232425262728293031323334353637383940 Sample No.

C

$\square$ hepcidin-20 $\square$ hepcidin-22 $\square$ hepcidin-25

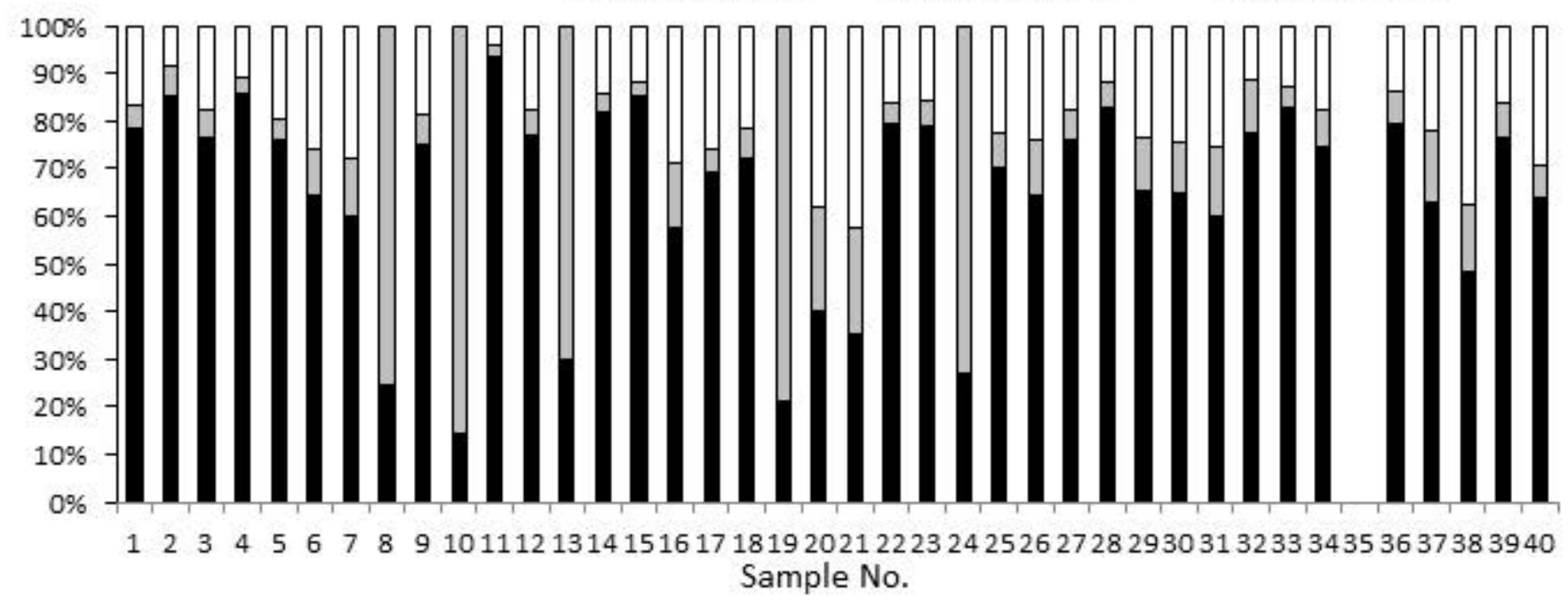


A

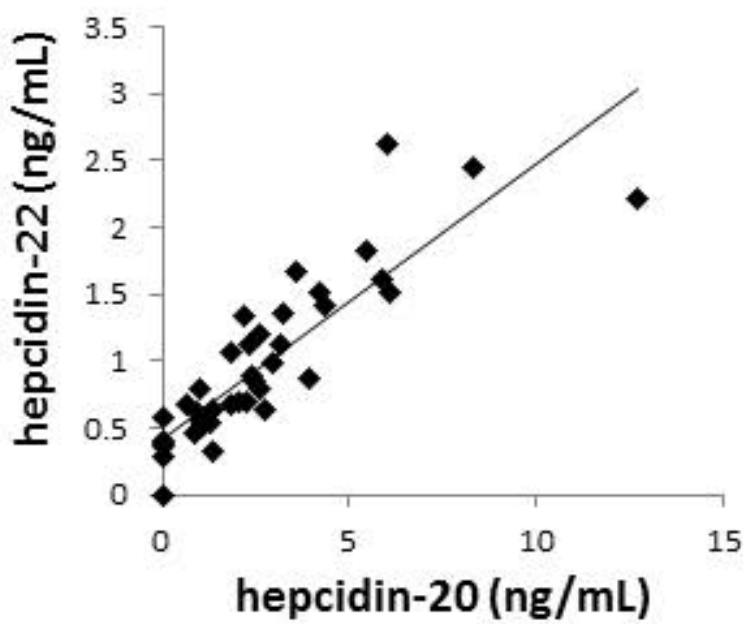

C

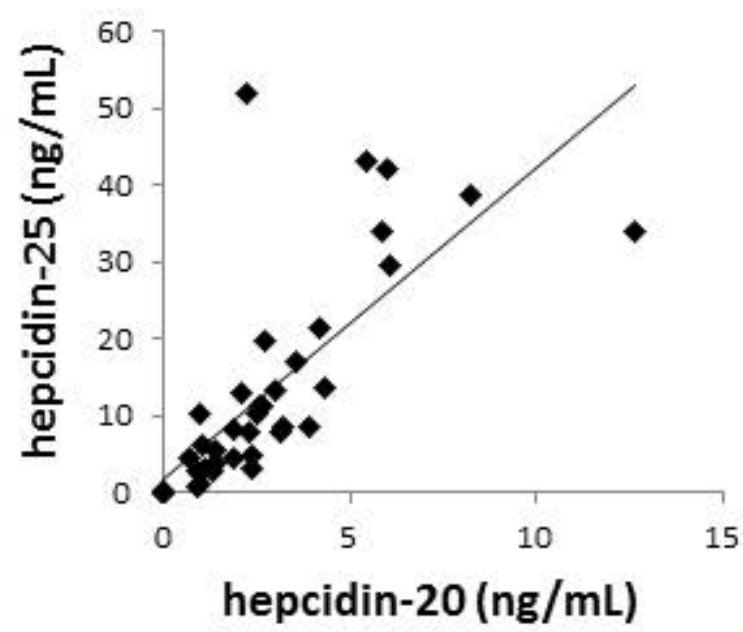

B

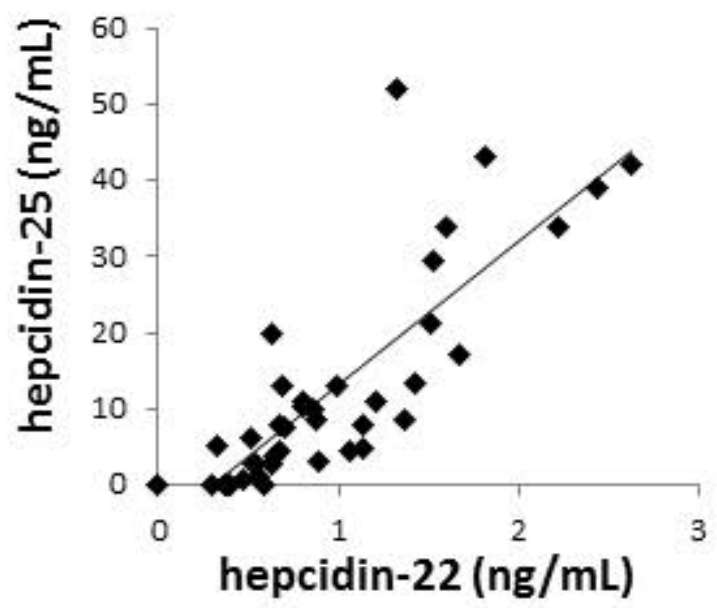


A

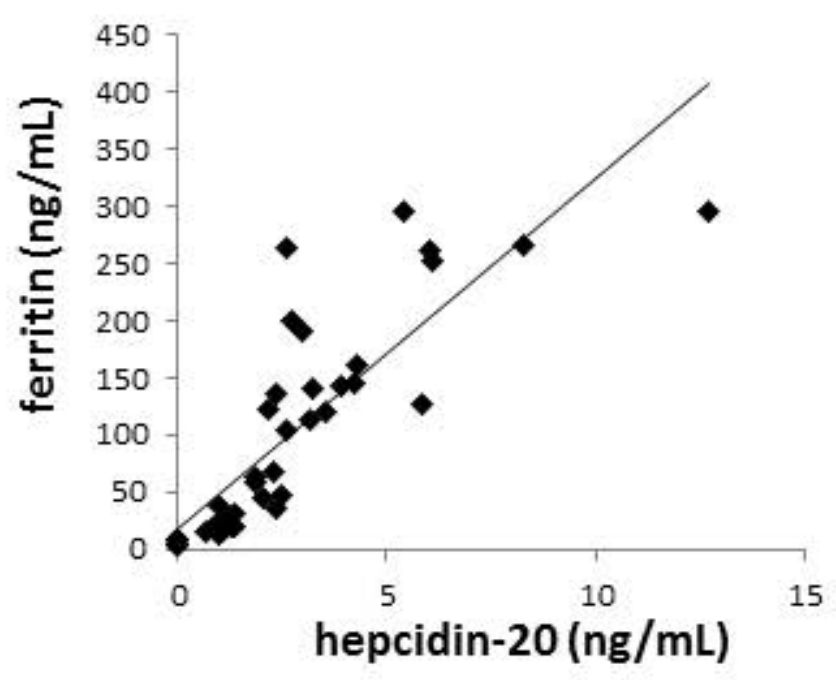

C

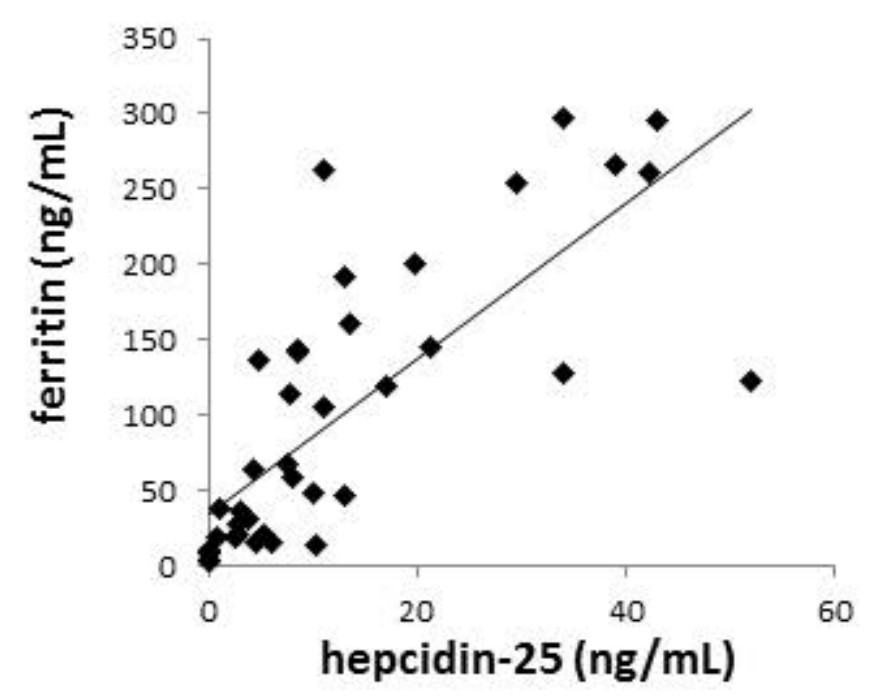

B

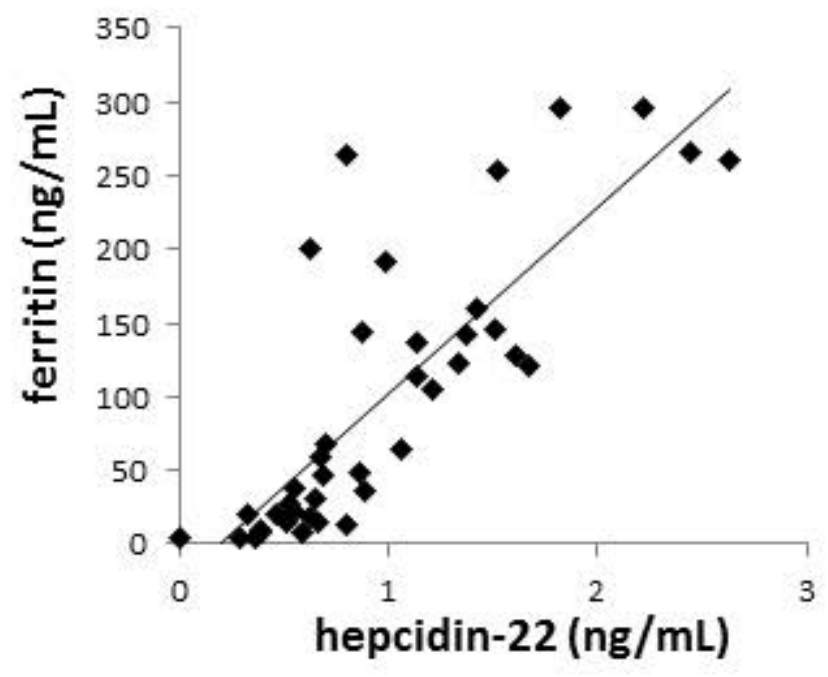


A

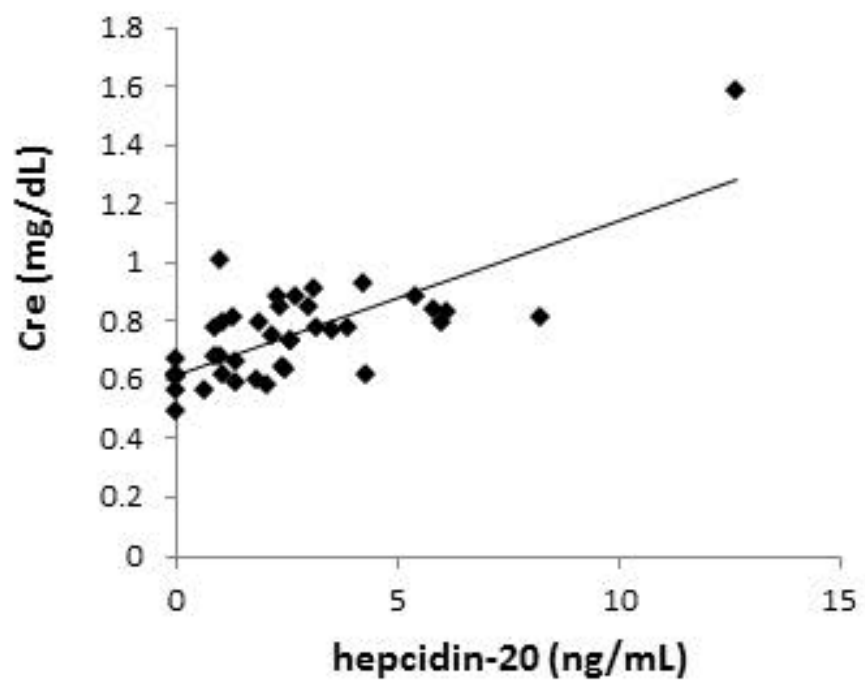

B

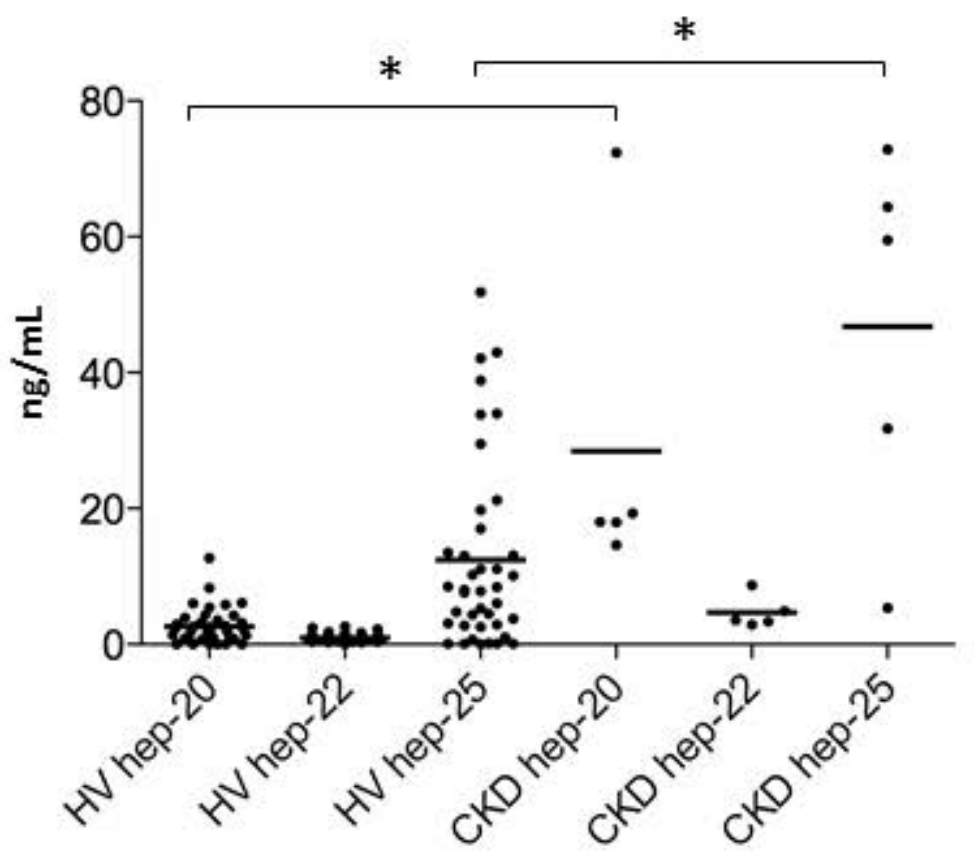


A

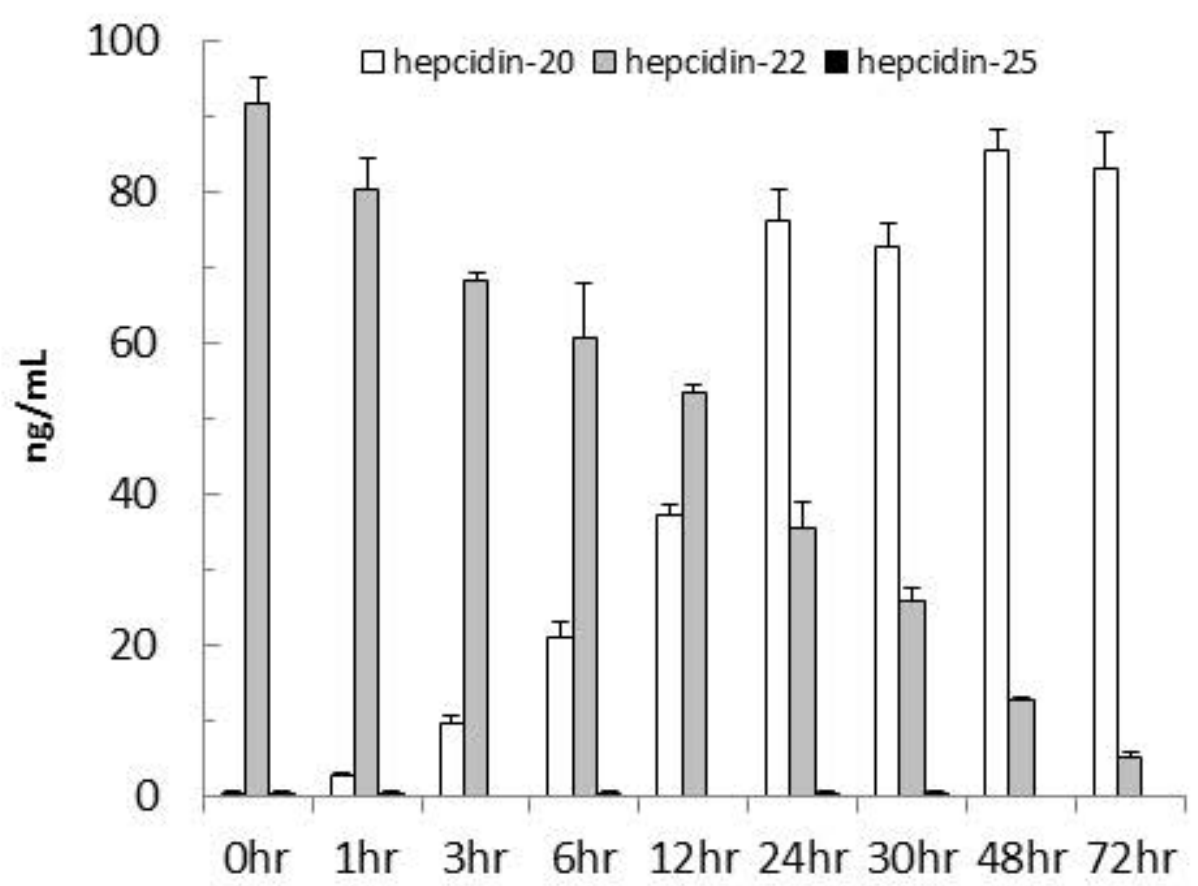

B

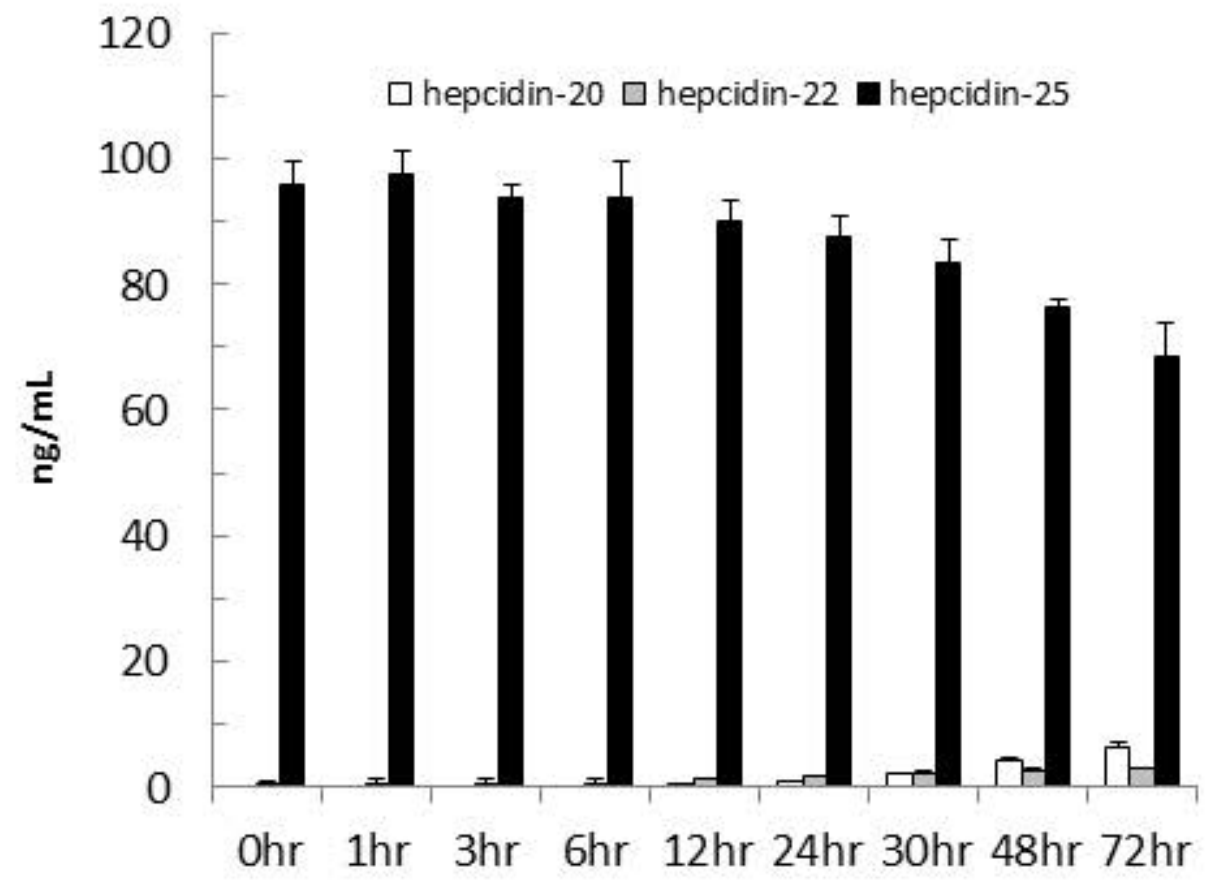


A

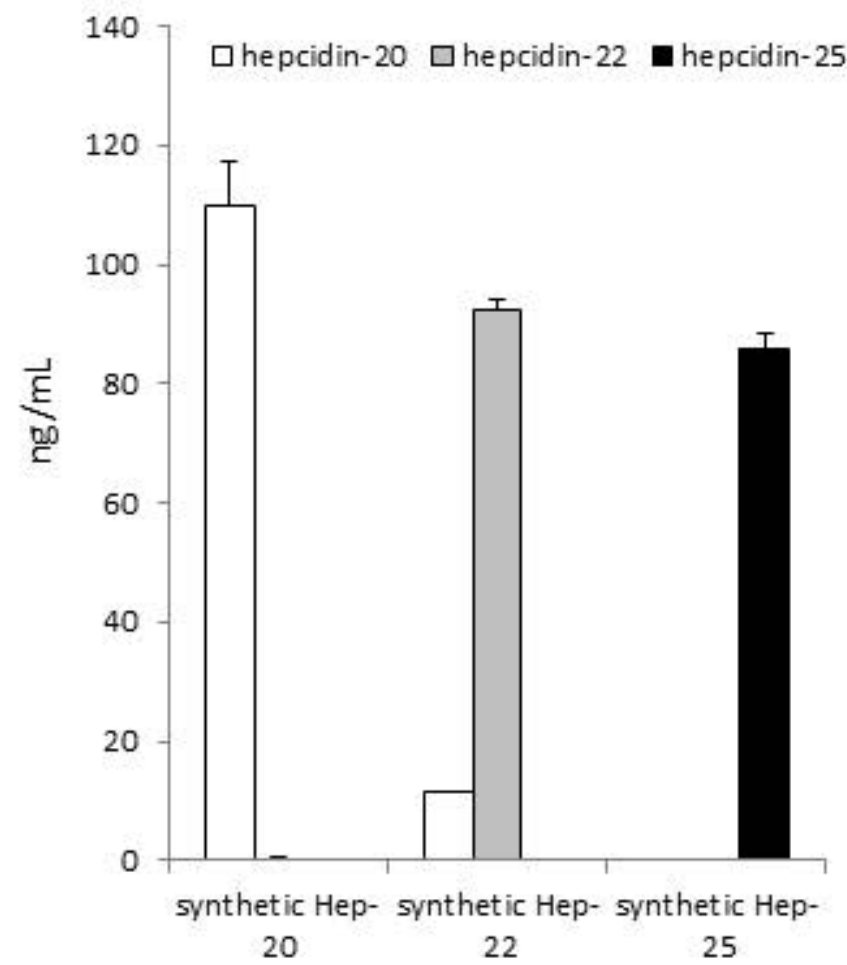

B

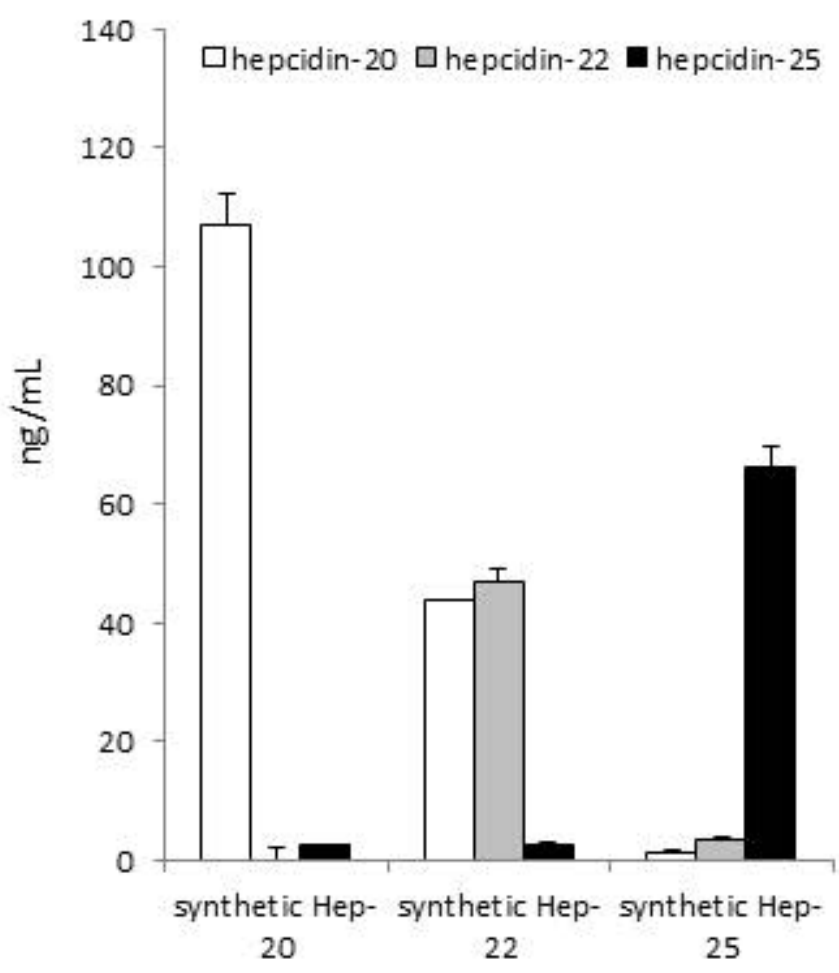

